\title{
Applied Anatomy for Botulinum Toxin Injection in Cosmetic Interventions
}

\author{
Ayman D'Souza ${ }^{1}$ (D) $\cdot$ Chew Lip $\mathrm{Ng}^{2}$ \\ Published online: 18 August 2020 \\ (C) The Author(s) 2020
}

\begin{abstract}
Purpose of Review To provide the reader with a clear overview of facial anatomy as it relates to injection of botulinum toxin. Recent Findings The review suggests the presentation of multiple combinations of facial musculature, with the forehead, glabellar, and nasal base areas as particular areas of variation. Differences in musculature result in different wrinkling patterns; with age, these changes first become apparent in the upper face, particularly in the forehead and glabellar area.

Summary Botulinum toxin is well suited to achieve the optimal outcome: it is popular, safe, and non-invasive and presents with few adverse effects. Though limited, when adverse effects do arise these are primarily related to poor injection technique, often fuelled by poor anatomical knowledge. For clinicians to achieve the best outcomes with botulinum toxin use, an understanding of the facial muscles' anatomy, actions, and interactions is key. This paper discusses the broad and intricate detail regarding the key target muscles of botulinum toxin, based on both literature review and cadaveric dissection carried out by the authors.
\end{abstract}

Keywords Botulinum toxin · Facial anatomy $\cdot$ Forehead region

\section{Introduction}

Botulinum toxin (BoNT) is a popular therapeutic and cosmetic drug, used for a wide variety of medical and cosmetic procedures. Botulinum toxin type A was used for 7,437,378 cosmetic procedures in the USA in 2018, a $3 \%$ rise from that in 2017, and $845 \%$ rise since 2000 (https:/www.plasticsurgery. org/documents/News/Statistics/2018/plastic-surgerystatistics-report-2018.pdf). It is both minimally invasive and fast-acting, resulting in high satisfaction rates, with $96 \%$ of patients indicating they would be happy to receive treatment again (https://www.statista.com/statistics/281434/

This article is part of the Topical collection on Use of Botulinum Toxin in Otorhinolaryngology

Ayman D'Souza

Chew Lip $\mathrm{Ng}$

chew_lip_ng@nuhs.edu.sg

Bromley, UK

2 Department of Otolaryngology-Head and Neck Surgery, Ng Teng Fong General Hospital, National University Health System, 1 Jurong East Street 21, Singapore 609606, Singapore satisfaction-among-patients-of-top-us-nonsurgical-cosmeticprocedures/). Similar trends are evident in the rest of the world.

As well as leading to unsatisfactory results, poor understanding of facial anatomy can lead to suboptimal outcomes and complications, which may present legal risks to clinicians. Whilst not a fatal mistake, this leads to severe and undesirable side effects for patients, with muscular paralysis lasting weeks to months; BoNT spread to nearby muscles can also cause delocalisation of effects [1].

In some cases, longer term complications can arise. However, there exists a general scarcity of systematic literature concerning the specific common adverse effects of cosmetic BoNT use [2••]. Clinicians with a clear knowledge of anatomy relating to target muscles during injection are likely to have lower complication rates.

\section{Concepts Underlying BoNT Use}

\section{Static and Dynamic Rhytids}

A rhytid, or wrinkle, is a crease in the skin and can range from a fine line to a deep furrow. Glabellar lines and nasolabial 
folds are examples of deep furrows, and early crow's feet or lateral periorbital rhytids are examples of fine lines. Rhytids can broadly be classified as dynamic or static. Dynamic rhytids are present only during muscular contraction, whilst static rhytids are present even without muscular activity. The progression of rhytid formation begins with fine lines and dynamic wrinkles, but over time with the effects of repetitive action combined with muscular thinning and ageingassociated skin changes, static deep rhytids form.

BoNT-induced weakening and atrophy of mimetic facial muscles are effective in reducing or obliterating dynamic rhytids but not as effective for static rhytids. In particular, deep static furrows require other treatment including dermal fillers and lasers or other energy devices for improvement. The effects of BoNT on dynamic rhytids have been hypothesised to be due to (i) weakening of the underlying mimetic muscles, whose movements lead to skin creasing; (ii) weakening of mimetic muscles that leads to impairment of lymphatic drainage from the skin in the area, leading to mild skin oedema and improvement in rhytid; and/or (iii) BoNT's reduction of sweat gland activity, leading to improved skin quality.

\section{Balancing of Opposite Muscular Forces}

Facial muscles are arranged to pull in opposing directions, maintaining a balance of forces between muscles in up-down, medial-lateral, and sphincteric opening-closing directions. When one muscle is weakened, the effects of the opposing muscle(s) go unopposed and antagonist action is accentuated. This can lead to desirable or undesirable consequences depending on the muscles and regions in question. Of particular clinical relevance are the groups of elevators and depressors in the periorbital and perioral regions (Table 1).

This is well represented by the elevation of the brows from the paralysis of the brow depressor muscles, which is in most instances a positive outcome; and the ptosis (an undesirable secondary outcome) that may ensue when the frontalis (the main brow elevator) is paralysed to treat horizontal forehead rhytids. As such, one rarely paralyses the frontalis without also weakening the brow depressors, to balance the opposing up and down forces. Therefore, as in a game of chess, the skilled BoNT injector must consider not just the current injection, but also several steps in advance. The understanding of this concept is of paramount importance in the use of BoNT.

\section{Upper Third of the Face}

The upper third of the face can be further subdivided into 3 muscle groups: the forehead, the glabellar muscles, and the periocular region. Each region exhibits specific changes that are directly influenced by the action of muscles in the area.

The forehead musculature is dominated by the frontalis muscle.

The frontalis muscle is a paired sheet-like muscle that originates from the galea aponeurotica at the level of the coronal suture (aponeurosis of the epicranius), and inserts into the deep layer of the skin of the eyebrow/forehead, with one belly on each half of the forehead. Its contraction lifts the eyebrow and also produces horizontal wrinkles in the forehead. Though not often recognised, the muscle has four distinct configuration patterns, which are responsible for the variability of wrinkling patterns of the forehead [3•]. These are:

- Type I full-form bellies, which join at the midline and cover the whole forehead, giving rise to straight lines across the entire forehead (Fig. 1a)

- Type II V-shaped bellies, separated in the midline by a Vshaped projection of the aponeurotic galea, creating gull wing-shaped lines on the forehead (Fig. 1b)

- Type III central form bellies, which join in the midline over the medial half of the orbital rims, but do not extend as laterally as type I bellies, creating a column of short lines in the middle of the forehead (Fig. 1c)

- Type IV lateral form bellies, separated medially by a vertical rectangular aponeurotic galea projection, occupying only the more lateral forehead areas, and creating two short lateral columns of lines on the forehead, with an unwrinkled middle (Fig. 1d)
Table 1 Elevators and depressor facial muscles

\begin{tabular}{lll}
\hline & Elevator muscles & Depressor muscles \\
\hline Periorbital & Frontalis & Corrugator supercilii \\
& & Procerus \\
& & Orbicularis oculi \\
Perioral & Levator labii superioris & Depressor supercilii \\
& Levator labii superioris alaeque nasi & Depressor anguli oris \\
& Zygomaticus major & Depressor labii inferioris \\
& Zygomaticus minor & Platysma \\
& Levator anguli oris & \\
\hline
\end{tabular}


Fig. 1 a-d The four configurations of the frontalis muscle. Muscles' most extensive configurations are outlined in blue dashed lines, with colour used for clarity. Areas devoid in muscle, for muscles of different configurations, are represented by white lines on a grey background, with black dashed borders

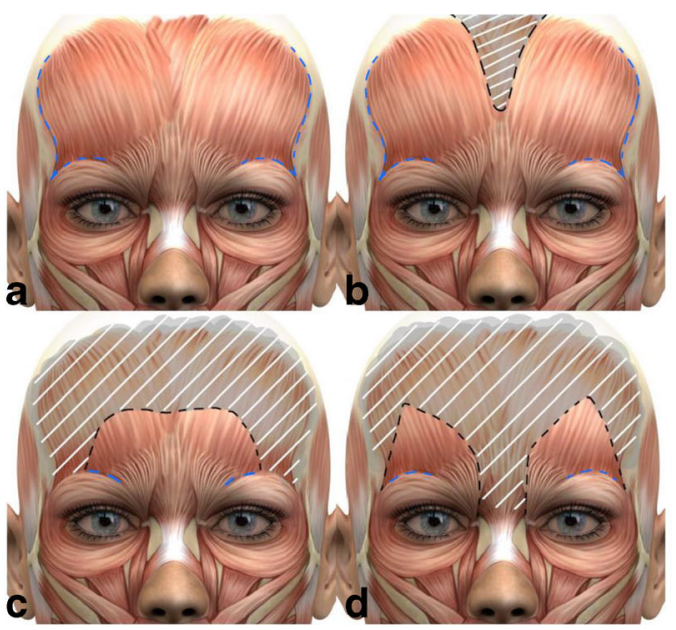

Areas devoid of muscle

During treatment of the frontalis, the configuration should be noted and sites of injections adjusted as required, to target areas with active muscles, achieve a good aesthetic outcome, and prevent asymmetry [3•]. Variations in muscle configuration create different patterns in wrinkling; the injector should be cognizant of these variations and avoid injecting areas deficient in muscle, which are neither useful nor create good outcomes for patients.

Weakening of the frontalis will lead to brow ptosis; to prevent this, frontalis injections often need balancing with injections to weaken the brow depressors. The injector must note the position of the brows and eyelids pre-injection and avoid exacerbating pre-existing brow ptosis and/or blepharoptosis and should explain the possibility of exacerbation of ptosis after injection.

The glabellar muscles comprise the corrugator supercilii, depressor supercilii, and procerus muscles. These muscles are primarily the depressors of the medial brow.

The corrugator supercilii contracts to pull the skin of the eyebrow downward and medially. It originates from the medial part of the superciliary arch and inserts into the skin over the middle of the eyebrow, penetrating the frontalis and/or orbicularis oculi muscles in doing so. It is thicker at the medial canthus than the midpupillary line, an important consideration for injectors [4]. Similar to the frontalis, it exists in different configurations. Three shapes have been identified, but unlike the frontalis, these do not always present symmetrically, creating 6 distinct wrinkle/ muscle patterns, three symmetrical and three asymmetrical. Symmetrical patterns are:

- Type I (fan-shaped) insertion, along the entire medial half of the eyebrow, and hockey stick-shaped wrinkling on the medial eyebrow area (Fig. 2a)

- Type II (rectangular) insertions in the intermediate third of the eyebrow creating parallel straight lines on the glabella (Fig. 2b)

- Type III (narrow ribbon) insertion medial section of the eyebrows creating a single vertical line on the glabella (Fig. 2c)

Asymmetrical patterns are seen as combinations of types I, II, and III, creating three further patterns.

- Type IV conformation combines one fan-shaped muscle and one rectangular, producing hockey stick shape and parallel straight lines.
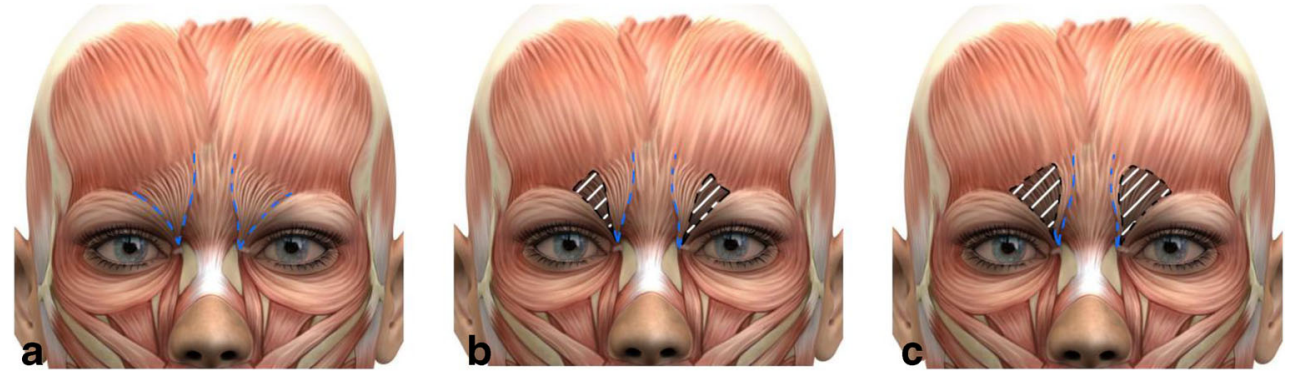

Fig. 2 a-c The three possible corrugator configurations. Muscles' most extensive configurations are outlined in blue dashed lines, with colour used for clarity. Areas devoid in muscle, for muscles of different configurations, are represented by white lines on a grey background, with black dashed borders 
- Type V combines fan-shaped with narrow ribbon on the other, creating a hockey stick-shaped line and single straight line.

- Type VI combines rectangular form and narrow ribbon contralaterally, resulting in several parallel straight lines on the glabella.

Injectors should be aware of this and avoid injecting areas deficient in muscle, noting that different injection locations may be necessary on respective sides of the face [3•].

The depressor supercilii, though sometimes considered part of the orbicularis oculi or corrugator, is a distinct entity [5], originating from the fascia covering the lower part of the nasal bone of the medial orbital rim, and inserting into the skin below the eyebrow in the intercanthal region, on the medial side of the bony orbit. Both muscles contract to form vertical rhytids, especially of the inner brow [6].

The procerus is pyramid-shaped and originates from both superficial and deep layers at the midline of the nasal bone and cartilage, and inserts into the skin of the lower forehead between the eyebrows and into the frontalis muscle belly. Most fibres form part of the hourglass-shaped superficial portion of the procerus, which is narrowest at the level of the medial palpebral ligament, spreading in a fan shape above and below this point. Patients with a wider procerus will present with wider fanning above and below, whilst a narrow procerus extends to the transverse part of the nasalis in a straight band before spreading into a narrow fan [7]. Its vertically oriented fibres contract to depress the medial eyebrow angle, also forming a transverse fold across the root of the nose and horizontal rhytids [6].

The major risk of BoNT injections in this region is blepharoptosis. This occurs when the toxin spreads to the levator palpebrae superioris, a lid elevator, leading to its paralysis. To prevent this, injections into the corrugator supercilii can be directed upwards, with a thumb placed on the superomedial orbital rim during injection to prevent diffusion of BoNT inferolaterally. When blepharoptosis occurs as a complication of BoNT, alpha-sympathomimetic eye drops can be used to stimulate the Mueller muscle, another lid elevator, to partially compensate for the paralysis of the levator palpebrae superioris.

The periocular region is dominated by the thin, superficial orbicularis oculi muscle (Fig. 3). This complex sphincteric muscle encompasses the eye and helps close the palpebral fissure. It has clearly defined subdivisions (orbital, palpebral, and lacrimal parts) which contract with different results. Cosmetic treatments focus on the orbital part, which originates from the medial palpebral ligament, runs around the eye in a complete ellipse shape, and inserts into the same location. Contraction of the orbicularis results in eye closure and also a downward pull of both medial and lateral brow. With age and muscle relaxation, "crow's feet" wrinkles appear in the

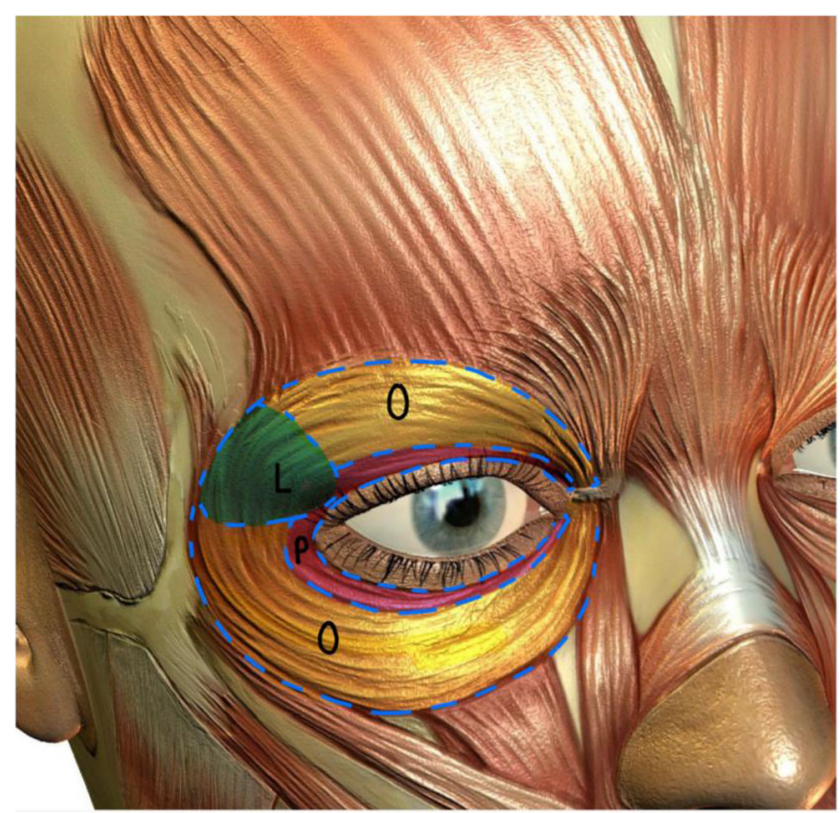

Fig. 3 The lacrimal (L), orbital (O), and palpebral (P) parts of the orbicularis oculi. Muscles' most extensive configurations are outlined in blue dashed lines, with colour used for clarity. Areas devoid in muscle, for muscles of different configurations, are represented by white lines on a grey background, with black dashed borders

corner of the eye (lateral canthus region). These rhytids are typically more pronounced than other facial wrinkling, possibly due to the lack of sebaceous glands in this area as compared with the forehead [8]. Though typical in normal ageing, wrinkling here occurs as early as patients' mid-20s, due to decreased skin elasticity after collagen and elastin loss, factors exacerbated by UV exposure and smoking [9]. During eyelid movement, contraction of the orbicularis oculi accentuates these wrinkles. The action of the zygomaticus muscles results in the elevation of lateral cheek tissues and further exaggerates the wrinkles.

Injecting the orbicularis in specific areas helps reduce periocular wrinkling and can also treat brow elevation. Due to the superficial nature of the muscle, we typically place small droplets of BoNT just subdermally $1 \mathrm{~cm}$ lateral to the lateral rim. Placement of a droplet below the lateral brow addresses the brow depressor function of the muscle, leading to lateral brow elevation and improvement of lateral hooding. A popular technique in Asia is the injection of a small droplet about $2 \mathrm{~mm}$ below the lower lid margin in the midpupillary line to weaken the sphincter function of the lower lid muscle, leading to a slight widening of the palpebral fissure and the appearance of a "bigger" eye.

\section{Special Features}

The frontalis elevates the brow. However, the procerus, corrugator supercilii, and depressor supercilii depress the 
medial brow, and the orbicularis oculi depresses the lateral brow [10]. In a BoNT brow lift, elevation occurs due to paralysis of brow depressor muscles [11]. This results in reduced opposition to the action of the frontalis, elevating the brow.

\section{Middle Third of the Face}

The nasalis muscle has a transverse portion and an alar portion.

The transverse portion of the nasalis originates from the maxilla lateral to the incisive fossa and inserts into the aponeurosis of the bridge of the nose. Its contraction compresses the nostrils and draws skin adjacent to the nose more medially, forming characteristic bunny lines, fine wrinkles which are especially visible in smiling or squinting, and exacerbated by overuse of the nasalis. This occurs naturally with ageing as the skin loses elasticity, but also results from transverse nasalis recruitment after BoNT administration to agonist muscles (without also targeting the nasalis), especially after treatment for glabellar folds or periocular wrinkles.

The alar portion of nasalis muscle contracts to lengthen the nose and dilate the nostrils. It originates from the outer surface of the maxilla over the lateral incisor and inserts into the greater alar cartilage.

The dilator naris anterior is responsible for nasal flaring, a healthy process which prevents the collapse of the nasal valve (alongside the alar part of the nasalis) during inspiration [12]. It originates from the upper lateral cartilage and alar portion of the nasalis and inserts into the skin of the caudal margin of the lateral and lateral alar crus. Flaring of the nostrils is a natural facial expression but over-contraction of the nasalis muscle may cause excessive flaring, especially during speech or smiling, causing embarrassment and distracting from desired facial expressions. BoNT treatment here is only effective and suitable for patients who are able to actively flare their nostrils, and undesirable side effects including lip asymmetry of upper lip lengthening may arise. https:/e-mastr.com/lessons/btxdilator-naris-nasal-flaring-injections/.

The levator labii superioris alaeque nasi (LLSAN; Fig. 4) contracts to dilate the nostrils and elevates the wing of the nose and upper lip, creating a snarling facial expression. It is often solely responsible for the emergence of a gummy smile appearance, or significantly exacerbates this phenomenon. It originates from the frontal process of the maxilla and inserts into the skin of the lateral part of the nostril and upper lip. A small proportion of its fibres extend to blend into the area of the procerus/depressor supercilii, and others extend to form the lateral superficial procerus [7]. The muscle comprises a superficial and deep layer; the superficial LLSAN descends on the levator labii superioris (LLS), and the deep LLSAN itself originates from the superficial layer and frontal process of the maxilla, before inserting between the levator anguli oris

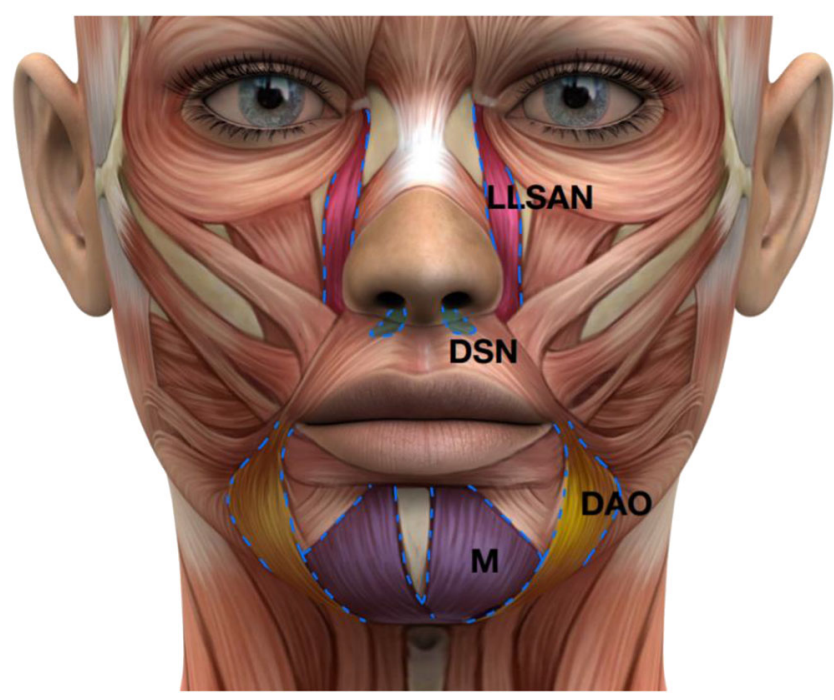

Fig. 4 Diagram of the face showing the following: levator labii superioris alaeque nasi (LLSAN), depressor septi nasi (DSN), depressor anguli oris (DAO), mentalis (M). Muscles' most extensive configurations are outlined in blue dashed lines, with colour used for clarity. Areas devoid in muscle, for muscles of different configurations, are represented by white lines on a grey background, with black dashed borders

and the orbicularis oris. In most people, the transverse nasalis receives some superficial LLSAN fibres [13].

The depressor septi nasi (DSN; Fig. 4) originates from the incisive fossa of the maxilla and inserts into the nasal septum and posterior aspect of the alar part of the nasalis. Its contraction depresses the nasal septum, draws the nose downwards, and decreases the size of the nostrils. It is powerful, and its pull on the nasal tip can overpower the effects of opposing muscles, creating an aesthetically undesirable "droopy" appearance to the tip of the nose. This is most obvious when smiling, but can present at rest too, and is exacerbated by ageing, and soft or excess nasal cartilage.

Three different DSN formations are seen. Type I DSN inserts fully and completely into the orbicularis oris, type II inserts into the periosteum and incompletely into the orbicularis oris, and type III shows no or a rudimentary depressor septi muscle [14]. The exact distribution of these types seems to vary in the population [15], but injectors should be cognizant of different conformations, or the possible lack of a depressor septi nasi (although these patients are unlikely to present with a droopy tip nose) [16].

\section{Lower Third of the Face}

The masseter is a major muscle of mastication. It is a thick, quadrilateral muscle that originates from the temporal process of the zygomatic bone and anterior two-thirds of the zygomatic arch, and passes posteroinferiorly to be inserted into the angle of the mandible and the lower half of the lateral ramus of the mandible [17]. This muscle can be hypertrophied in 
conditions such as bruxism and renders a squarish shape to the lateral jawline, which is a masculine feature. This can be a positive point in men and in cultures where a strong jaw is seen as aesthetically pleasing. In Asian cultures, a squared jawline in women is not viewed as aesthetically pleasing, and a V-shaped jawline is preferred. As such, BoNT injection into the masseter to induce atrophy and reduction in its bulk is commonly performed. Masseteric injection is also performed to weaken the masseter in maxillofacial conditions like bruxism to reduce the erosive forces on the dentition.

Injections are usually performed within the lower third of the bulk of the masseter. The patient is asked to clench her teeth; the muscle is palpated and divided into 4 quadrants below a line drawn from the angle of the mouth to the ear lobe. One injection is administered into each of these quadrants. The injections should be performed deep into the muscle bulk. Superficial and anterior injections can lead to diffusion to the thin risorius muscle which lies just anterior to the masseter. The risorius lies $0-15 \mathrm{~mm}$ anterior to the anterior border of the masseter, arising from the SMAS and parotidomasseteric fascia and inserts into the modiolus. It pulls the angle of the mouth superolaterally; inadvertent paralysis of the risorius can lead to asymmetry when smiling.

The orbicularis oris muscle is a complex, multi-layered muscle which attaches through a thin SMAS to the dermis of the upper lip and lower lip and serves as an attachment site for many other facial muscles around the oral region [18]. It is without bony or tendinous origin and is treated as a single anatomical muscle but comprises multiple parts which act and interact with surrounding facial muscles independently [19]. Its deep "archaic" part has constrictor fibres which contribute to the mouth's sphincter-like action, whilst superficial retractor fibres (which control facial expression and speech-associated precise lip movement) are those typically targeted by BoNT treatment [20]. Interactions with other facial muscles allow pressure creation in the mouth for speech [21], and it also facilitates swallowing, mastication, lip puckering, and sucking [22, 23].

With age, orbicularis oris contraction produces perioral wrinkling, more visible in women than that in men, partially because this muscle is anchored 1.5 times closer to the dermis in women than that in men [24]. BoNT delays wrinkle reappearance after filler use and enhances the action of these treatments, by preventing pursing mouth actions and also giving a slight augmentation appearance to the lips. However, this treatment is unsuitable for those who rely on this muscle's actions, such as scuba divers or wind instrument players, and patients who use straws, as they are likely to temporarily lose this ability (https:// www.the-dermatologist.com/article/6858).

The depressor anguli oris (DAO; Fig. 4) is a paired muscle which depresses the mouth corner to form a frowning expression. It is continuous with other muscles at both its origin and insertion: the platysma at its origin, and the orbicularis oris and risorius at its insertion. It arises from the oblique line of the mandible and inserts into the modiolus. Where it overlaps with surrounding muscles, it has fibres continuous with the caninus, and in some cases contributes to the transversus menti, with fibres which join the two muscles of the pair.

With age and decreased elasticity, the increased pull of this muscle on the mouth can generate an unhappy and discontent expression, with a droopy mouth corner associated with sadness and even anger in extreme cases [25, 26]. BoNT injection is an effective procedure to counter and diminish the effect of the depressor anguli oris; however, the nearby orbicularis oris and depressor labii inferioris present hazards, so low, superficial, external injections are preferred to avoid adverse effects [27].

The mentalis (M; Fig. 4) is a paired central muscle of the lower lip, found in the tip of the chin. Its fibres originate from the incisive fossa on the alveolar process of the mandible in the region of the lateral incisor, and radiate and insert into the skin and soft tissue of the chin. Upper lateral fibres are interlaced with the origins of the incisivus labii inferioris, continuing in some patients into middle and lower portion mentalis regions, and all upper fibres intermingle with the lower orbicularis oris [28]. Contraction of the muscle draws the chin upwards and inwards, also raising the central portion of the lips, which can allow "pouting" in conjunction with the orbicularis oris. Contraction also causes an external wrinkling of the chin, due to the muscles' insertions into the skin, contributing to a doubtful, indecisive, or displeased facial expression, which BoNT treatment aims to remove. The injection points are $1 \mathrm{~cm}$ lateral from the midline on each side. Injecting too laterally may lead to paralysis of the depressor labii inferioris, leading to ptosis of the lower lip and abnormal mouth movement and smile.

\section{Neck Region}

The platysma is a wide, flat, superficial paired muscle, dividing into mandibular, labial, and modiolar (nodular) parts in the mouth area. The muscle originates from skin and fascia over the lower neck and the upper lateral chest and inserts on the inferior border of the mandible and into the skin over the lower face and angle of the mouth. Upon contraction, the platysma causes the skin of the lower face and mouth to become depressed and wrinkled, drawing lips inferiorly, and contributes to forced depression of the mandible. Males tend to present with bulkier, well-defined platysma, whilst females are more likely to have thinner platysma [29]. In time, the muscle shortens and thins [29], causing the skin lying above it to form vertical bands and wrinkles. The platysma is deficient in the midline. The medial borders of the platysma on both sides form the vertically oriented platysmal bands, a common sign of ageing. In most Caucasians, the medial platysmal fibres do not decussate, whilst in Asians, $85 \%$ of medial fibres are interlaced at the submental region [30]. This 
may explain the lower incidence of the "gobbler neck deformity" in Asians.

Platysmal bands can be addressed by injecting small amounts of BoNT directly into the bands at 1.5-2-cm intervals from the chin to the clavicle. A "microbotox" technique has been described to address horizontal neck lines in which many small droplets of diluted BoNT are injected with a very fine needle (32 gauge) into the subdermal layer at $0.5-1-\mathrm{cm}$ intervals over the entire platysma to both deep and fine horizontal lines in the neck.

\section{Conclusion}

BoNT injections are meant to be directed and precise, targeting specific muscles. Understanding the anatomy and functions of the muscles to target is key to the effective use of BoNT. In this article, we have described the anatomy of the commonly targeted muscles of the head and neck and described select clinical caveats in administering BoNT to these muscles.

\section{Compliance With Ethical Standards}

Conflict of Interest The authors declare that they have no conflict of interest.

Human and Animal Rights and Informed Consent This article does not contain any studies with human or animal subjects performed by any of the authors.

Open Access This article is licensed under a Creative Commons Attribution 4.0 International License, which permits use, sharing, adaptation, distribution and reproduction in any medium or format, as long as you give appropriate credit to the original author(s) and the source, provide a link to the Creative Commons licence, and indicate if changes were made. The images or other third party material in this article are included in the article's Creative Commons licence, unless indicated otherwise in a credit line to the material. If material is not included in the article's Creative Commons licence and your intended use is not permitted by statutory regulation or exceeds the permitted use, you will need to obtain permission directly from the copyright holder. To view a copy of this licence, visit http://creativecommons.org/licenses/by/4.0/.

\section{References}

Papers of particular interest, published recently, have been highlighted as:

- Of importance

• Of major importance

1. Bai L, Peng X, Liu Y, Sun Y, Wang X, Wang X, et al. Clinical analysis of 86 botulism cases caused by cosmetic injection of botulinum toxin (BoNT). Medicine (Baltimore). 2018;97(34):e10659. https://doi.org/10.1097/MD.0000000000010659.
2.• Yiannakopoulou E. Serious and long-term adverse events associated with the therapeutic and cosmetic use of botulinum toxin. Pharmacology. 2015;95(1-2):65-9. https://doi.org/10.1159/ 000370245. This study highlights the adverse effects which can occur when botulinum toxin is used in an unchecked or unsuitable manner.

3. Abramo AC, Do Amaral TP, Lessio BP, De Lima GA. Anatomy of forehead, glabellar, nasal and orbital muscles, and their correlation with distinctive patterns of skin lines on the upper third of the face: reviewing concepts. Aesthet Plast Surg. 2016;40(6):962-71. https://doi.org/10.1007/s00266-016-0712-z. Findings from this study highlighted the four configurations of frontalis muscle and married areas of musculature with the common presenting wrinkling patterns of the forehead.

4. Hwang K, Lee JH, Lim HJ. Anatomy of the corrugator muscle. J Craniofac Surg. 2017;28(2):524-7. https://doi.org/10.1097/SCS. 0000000000003304.

5. Daniel RK, Landon B. Endoscopic forehead lift: anatomic basis. Aesthet Surg J. 1997;17(2):97-104. https://doi.org/10.1016/S1090820X(97)80070-2.

6. Macdonald MR, Spiegel JH, Raven RB, Kabaker SS, Maas CS. An anatomical approach to glabellar rhytids. Arch Otolaryngol Head Neck Surg. 1998;124(12):1315-20. https://doi.org/10.1001/ archotol.124.12.1315.

7. Hur MS. Anatomical relationships of the procerus with the nasal ala and the nasal muscles: transverse part of the nasalis and levator labii superioris alaeque nasi. Surg Radiol Anat. 2017;39(8):865-9. https://doi.org/10.1007/s00276-017-1817-z.

8. Tamatsu Y, Tsukahara K, Sugawara Y, Shimada K. New finding that might explain why the skin wrinkles more on various parts of the face. Clin Anat. 2015;28(6):745-52. https://doi.org/10.1002/ca. 2257.

9. Lee JY, Kim YK, Seo JY, et al. Loss of elastic fibers causes skin wrinkles in sun-damaged human skin. J Dermatol Sci. 2008;50(2): 99-107. https://doi.org/10.1016/j.jdermsci.2007.11.010.

10. Frankel AS, Kamer FM. Chemical browlift. Arch Otolaryngol Head Neck Surg. 1998;124(3):321-3. https://doi.org/10.1001/ archotol.124.3.321.

11. Ahn MS, Catten M, Maas CS. Temporal brow lift using botulinum toxin A. Plast Reconstr Surg. 2000;105(3):1129-39. https://doi.org/ 10.1097/00006534-200003000-00046.

12. Sasaki CT, Mann DG. Dilator naris function: a useful test of facial nerve integrity. Arch Otolaryngol. 1976;102(6):365-7. https://doi. org/10.1001/archotol.1976.00780110077009.

13. Hur MS, Hu KS, Park JT, Youn KH, Kim HJ. New anatomical insight of the levator labii superioris alaeque nasi and the transverse part of the nasalis. Surg Radiol Anat. 2010;32(8):753-6. https://doi. org/10.1007/s00276-010-0679.

14. Rohrich RJ, Huynh B, Muzaffar AR, Adams WP Jr, Robinson JB Jr. Importance of the depressor septi nasi muscle in rhinoplasty: anatomic study and clinical application. Plast Reconstr Surg. 2000;105(1):376-88. https://doi.org/10.1097/00006534200001000-00059.

15. Farahvash MR, Ebrahimi A, Farahvash B, Farahvash Y. Anatomic and anthropometric analysis of 72 lower lateral nasal cartilages from fresh Persian (Iranian) cadavers. Aesthet Surg J. 2012;32(4): 447-53. https://doi.org/10.1177/1090820X12442084.

16. Barbosa MV, Nahas FX, Ferreira LM. Anatomy of the depressor septi nasi muscle: the basis for correction of deformities of the nose/lip junction. J Plast Surg Hand Surg. 2013;47(2):102-5. https://doi.org/10.3109/2000656X.2012.741526.

17. Bui AT, Widmer CG. Anatomical architecture of the human masseter muscle. J Dent Res. 1996;75:1795.

18. Ghassemi A, Prescher A, Riediger D, Axer H. Anatomy of the SMAS revisited. Aesthet Plast Surg. 2003;27(4):258-64. 
19. Vinkka-Puhakka H, Kean MR, Heap SW. Ultrasonic investigation of the circumoral musculature. J Anat. 1989;166:121-33.

20. Nicolau PJ. The orbicularis oris muscle: a functional approach to its repair in the cleft lip. Br J Plast Surg. 1983;36(2):141-53.

21. Green JR, Moore CA, Higashikawa M, Steeve RW. The physiologic development of speech motor control: lip and jaw coordination.

22. Park JS, Oh DH, Chang MY. Effect of expiratory muscle strength training on swallowing-related muscle strength in communitydwelling elderly individuals: a randomized controlled trial. Gerodontology. 2017;34(1):121-8.

23. Regalo SC, Vitti M, Moraes MT, Semprini M, de Felício CM, de Mattos MG, et al. Electromyographic analysis of the orbicularis oris muscle in oralized deaf individuals. Braz Dent J. 2005;16(3):237-42.

24. Paes EC, Teepen HJ, Koop WA, Kon M. Perioral wrinkles: histologic differences between men and women. Aesthet Surg J. 2009;29(6):467-72. https://doi.org/10.1016/j.asj.2009.08.018.

25. Perkins SW. The corner of the mouth lift and management of the oral commissure grooves. Facial Plast Surg Clin North Am. 2007;15:471-6 vii.
26. Goldman A, Wollina U. Elevation of the corner of the mouth using botulinum toxin type a. J Cutan Aesthet Surg. 2010;3:145-50.

27. Raspaldo H, Niforos FR, Gassia V, et al. Lower-face and neck antiaging treatment and prevention using onabotulinumtoxin $\mathrm{A}$ : the 2010 multidisciplinary Fr.

28. Hur MS, Kim HJ, Choi BY, Hu KS, Kim HJ, Lee KS. Morphology of the mentalis muscle and its relationship with the orbicularis oris and incisivus labii inferioris muscles. J Craniofac Surg. 2013;24(2): 602-4. https://doi.org/10.1097/SCS.0b013e318267bcc5.

29. Hwang K, Kim JY, Lim JH. Anatomy of the platysma muscle. J Craniofac Surg. 2017;28(2):539-42. https://doi.org/10.1097/SCS. 0000000000003318.

30. Kim H, Hu K, Kang M, Chung I, Hwang K. Decussation patterns of the platysma in Koreans. Br J Plast Surg. 2001;54(5):400-2.

Publisher's Note Springer Nature remains neutral with regard to jurisdictional claims in published maps and institutional affiliations. 